\title{
Modeling, Dynamics and Control of Spacecraft Relative Motion in a Perturbed Keplerian Orbit
}

\author{
Mohamed Okasha* \\ Department of Mechanical Engineering, IIUM Univ., Kuala Lumpur 50725, Malaysia
}

\author{
Brett Newman** \\ Department of Mechanical and Aerospace Engineering, Old Dominion University, Norfolk, VA 23529, USA
}

\begin{abstract}
The dynamics of relative motion in a perturbed orbital environment are exploited based on Gauss' and Cowell's variational equations. The inertial coordinate frame and relative coordinate frame (Hill frame) are used, and a linear high fidelity model is developed to describe the relative motion. This model takes into account the primary gravitational and atmospheric drag perturbations. Then, this model is used in the design of a navigation, guidance, and control system of a chaser vehicle to approach towards and to depart from a target vehicle in proximity operations. Relative navigation uses an extended Kalman filter based on this relative model to estimate the relative position/velocity of the chaser vehicle with respect to the target vehicle. This filter uses the range and angle measurements of the target relative to the chaser from a simulated LIDAR system. The corresponding measurement models, process noise matrix, and other filter parameters are provided. Numerical simulations are performed to assess the precision of this model with respect to the full nonlinear model. The analyses include the navigation errors and trajectory dispersions.
\end{abstract}

Key words: Control, Dynamics, Keplerian Orbit, Relative Motion

\section{Introduction}

The autonomous rendezvous and docking of a satellite in orbit is one of the most essential technologies for future autonomous space transportation missions, such as International Space Station supply and repair, automated inspection, servicing, and assembly of space systems. However, in most space programs, the rendezvous and docking is currently achieved by manual operations. Autonomous proximity operations are required for a large number of future mission concepts, but they cannot be achieved routinely at present. The interest in autonomous rendezvous and proximity operations has increased with the recent demonstration of XSS-11, Demonstration of Autonomous Rendezvous Technology (DART), and Orbital Express. Autonomous rendezvous and proximity operations have also been demonstrated by Japanese EST-VII and the Russian Progress vehicles. In addition, future missions to the ISS will require autonomous rendezvous and proximity operations $[1,2]$.

Many relative motion modeling and control strategies have been designed using the linearized Clohessy-Wiltshire (CW) equations to describe the relative motion between satellites. The CW equations are valid, if two conditions are satisfied: (1) the distance between the chaser and the target is small compared with the distance between the target and the center of the attracting planet, and (2) the target orbit is near circular [3]. The CW equations do not include any disturbance forces- for example, gravitational perturbations and environmental forces (solar radiation pressure and atmospheric drag). Alternative linear equations that have been used in the literature to model the relative motion are
This is an Open Access article distributed under the terms of the Creative Commons Attribution Non-Commercial License (http://creativecommons.org/licenses/bync/3.0/) which permits unrestricted non-commercial use, distribution, and reproduction in any medium, provided the original work is properly cited. ** Professor 
the Tschauner-Hempel (TH) equations [4], which generalize the CW equations and are similar to them in their derivation and types of applications. Tschauner and Hempel derived theses equations from the viewpoint from rendezvous of a spacecraft with an object in an elliptical orbit. They found complete solutions for elliptical orbits, in terms of the eccentric anomaly. This advancement was followed by additional papers that presented the complete analytical solution explicit in time, expanding the state transition matrix in terms of eccentricity [5-11]. These solutions are used to analyze the relative motion between the chaser and the target vehicles in the relative frame of motion more efficiently and rapidly than solving the exact nonlinear differential equations in the inertial coordinate system. However, the TH equations do not take into account any perturbation forces, though they have significant effects on the satellite relative motion.

As an attempt to overcome the previous limitations of the CW and TH models, this paper proposes an innovative linear model that includes both $J_{2}$ perturbation that reflects the Earth oblateness effect and atmospheric drag perturbation in the Cartesian coordinates orbital frame with little complication. Especially in low Earth orbits (LEOs), these perturbations have deep influence on the relative dynamics. Their inclusion in the linear model can sensibly increase the performance of the linear filters, which allows greater insight of satellite relative motion and provides an opportunity to investigate alternative feedback control strategies for the proximity operations.

This paper uses an extended Kalman filter formulation to estimate the relative position/velocity of the chaser vehicle, by utilizing the range and angle measurements from a simulated LIDAR system [12-16]. The Kalman filter basically consists of two main stages. The first stage is the propagation stage where the states are propagated numerically based on the proposed linear model. The second stage comes in when the measurements from the LIDAR system are available, and it is used to update the states from the first stage. The corresponding measurement models, process noise matrix, and other filter parameters are provided. Thrusters are assumed for translation control. The effects of the navigation filter and control algorithms are included in the analysis.

The objective of this paper is as follows: (1) develop a linearized high fidelity model for relative motion in a perturbed orbit, (2) design a navigation filter that can determine the relative position/velocity between target and chaser vehicles and support closed-loop proximity operations and maneuvers, and (3) design a control system for the chaser vehicle either to approach towards or to depart from a target vehicle in proximity operations in a general perturbed orbit.

The analysis in the current paper is summarized as follows. First, Section 2 presents the dynamic equations of relative motion for the chaser with respect to the target in a general perturbed orbit, based on Gauss' and Cowell's variational equations. These equations of motion are developed in the inertial coordinate frame, as well as in the relative coordinate frame. In Section 3, a linear high fidelity relative motion model is derived to describe the relative motion in proximity operations, by taking into account the gravitational $J_{2}$ perturbation and environmental atmospheric drag perturbation. In Section 4, the relative navigation using the extended Kalman filter and the controller design are presented based on this linear high fidelity model for relative motion. In Section 5, the accuracy and performance of the relative navigation and controller are illustrated through different numerical examples, and comparisons are made with the true nonlinear model. Finally, in Section 6 conclusion is presented and suggestions are made for future work.

\section{Relative motion dynamics}

Consider an Earth-centered inertial (ECI) frame with orthonormal basis $\left\{i_{X}, i_{Y}, i_{Z}\right\}$. The vectors $i_{X}$ and $i_{Y}$ lie in the equatorial plane, with $i_{X}$ coinciding with the line of equinoxes and $i_{Z}$ passing through the North Pole. Relative motion is conveniently described in a Local-Vertical-Local-Horizontal (LVLH) frame that is attached to the target spacecraft as shown in Fig. 1 . This frame has basis $\left\{i_{X}, i_{Y}, i_{Z}\right\}$, with $i_{X}$ lying along the radius vector from the Earth's center to the spacecraft, $i_{Z}$ coinciding with the normal to the plane defined by the position/velocity vectors of the target spacecraft, and $i_{Y}=i_{Z} \times i_{X}$. The LVLH frame rotates with the angular velocity $\omega$, and its current orientation with respect to the ECI frame is given by the 3-1-3 direction cosine matrix comprising right ascension of ascending node $\Omega$, inclination $i$, perigee argument $\omega$ plus true anomaly $f$ (Fig. 2 ). The angular velocity can be also expressed in terms of orbital elements and their rates.

Let the position of the chaser vehicle in the target's LVLH frame be denoted by

$\boldsymbol{\rho}=x i_{x}+y i_{y}+z i_{z}$, where $x, y$, and $z$ denote the components of the position vector along the radial, transverse, and outof-plane directions, respectively. Then, the most general equations modeling relative motion are given by

$$
\ddot{\boldsymbol{\rho}}=\left[\mathbf{f}_{c}\right]^{\mathrm{LVLH}}-\left[\mathbf{f}_{t}\right]^{\mathrm{LVLH}}-2 \boldsymbol{\omega} \times \dot{\boldsymbol{\rho}}-\boldsymbol{\omega} \times(\boldsymbol{\omega} \times \boldsymbol{\rho})-\dot{\boldsymbol{\omega}} \times \boldsymbol{\rho}
$$


where $\left[\mathbf{f}_{c}\right]^{\mathrm{LVLH}}$ and $\left[\mathbf{f}_{t}\right]^{\mathrm{LVLH}}$ are the external acceleration forces acting on the chaser and the target, respectively, in the LVLH frame of the target vehicle. $\left(\left(C^{\circ}\right)\right)$ and $\left(\left(^{\circ}\right)\right)$ denote the first and second derivatives with respect to time.

It is assumed that the external forces arise due to two basic groups of forces defined by the following equation.

$$
\mathbf{f}=\left(\mathbf{f}_{\mathrm{g}}+\mathbf{f}_{\mathrm{a}}+\mathbf{f}_{\mathrm{c}}\right)+\mathbf{f}_{\mathrm{w}}
$$

The first group of forces is due to gravitational effects, $\mathbf{f}_{\mathrm{g}}$ atmospheric drag, $\mathbf{f}_{a}$, and control, $\mathbf{f}_{c}$. Since the Earth is not perfectly spherical, more accurate gravity models exist that take into account the Earth's irregular shape. One irregularity that has a significant influence on space missions is the Earth's bulge at the equator. This phenomenon is captured in the $J_{2}$ gravity model $[17,18]$. The second group of forces, $\mathbf{f}_{w}$, is considered to be small forces due to the gravity fields of other planets, solar pressure, or venting which also perturbs the spacecraft's motion. These small forces are grouped together and modeled as normally distributed random variables with zero mean [14].

In the literature, the most popular methods to model the spacecraft orbit are known as Cowell's method and Gauss' method [16, 17]. The Cowell's method is basically defined by specifying the position $(\boldsymbol{R})$ and velocity $(\boldsymbol{V})$ vectors of the spacecraft in the inertial coordinate frame, while Gauss' method is defined by an equivalent set of elements called orbital elements (a, e, i, $\Omega, \omega, \mathrm{f}$ ) which correspond to the semi-major axis, eccentricity, inclination, right ascension of the ascending node, argument of periapsis, and true anomaly as shown in Fig. 2.

Table 1 summarizes the dynamic equations that are used to describe these methods. [.] ${ }^{\mathrm{I}}$ and [.] ${ }^{L V L H}$ denote that the forces are defined in the inertial and LVLH coordinate frames, respectively; $\mu$ and $R_{\oplus}$ are the Earth gravitational parameter and the radius of the Earth, respectively; the terms $R$ and $V$ refer to the magnitude of the position/velocity vectors, respectively; the quantity $H$ denotes the magnitude of the angular momentum vector

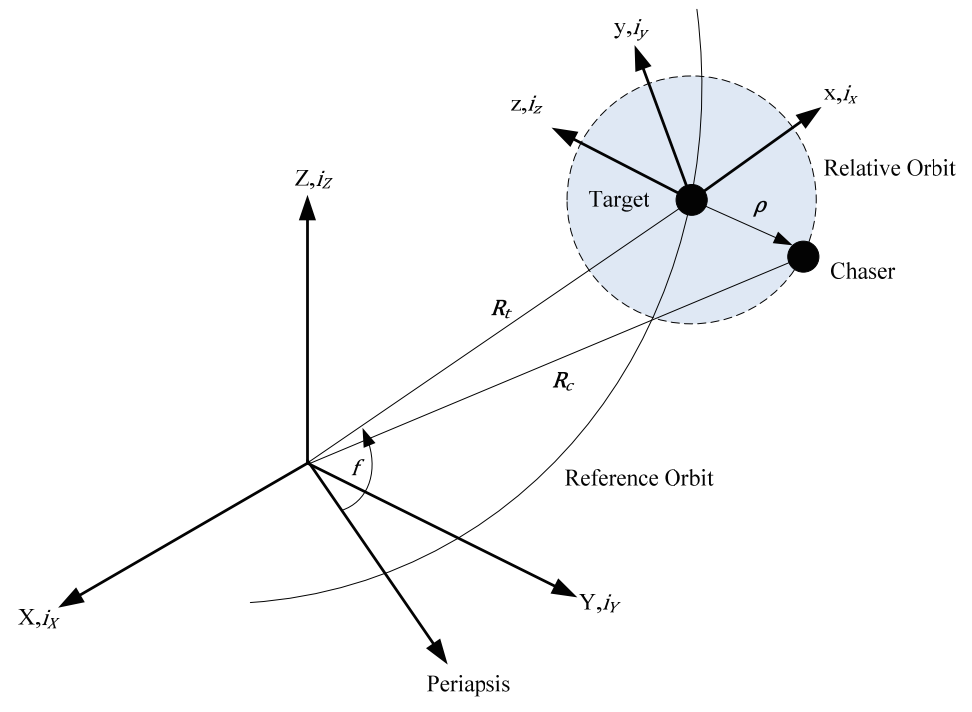

Fig. 1. Relative Motion Coordinates
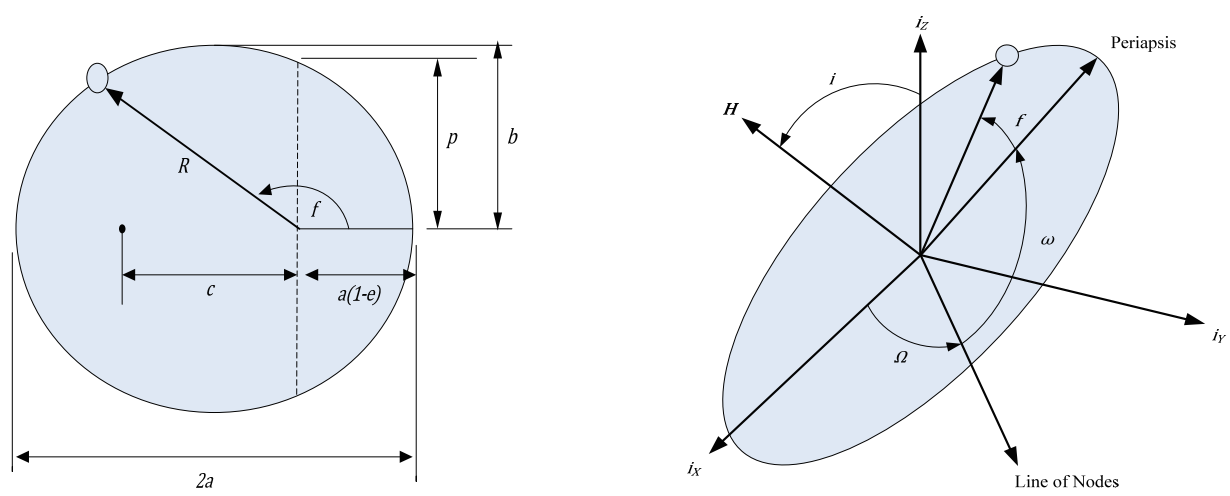

Fig. 2. Orbital Elements 
defined by $\boldsymbol{H}=\boldsymbol{R} \times \boldsymbol{V} ; X, Y$, and $Z$ are the components of the spacecraft position vector; $C_{D}$ is the atmospheric drag coefficient; $A$ denotes the spacecraft cross sectional area; $m$ is the spacecraft mass; and $\rho$ is the atmospheric density. Exponential model is used to describe the Earth atmospheric density. This model and its corresponding parameters are defined in Vallado [17].

In order to use the generalized relative dynamic model defined by Equation (1), the angular velocity vector, $\omega$, and the angular acceleration vector, $\dot{\omega}$, of the LVLH frame with respect to the ECI frame needs to be determined. Table 2 summarizes the equations that can be used to compute these vectors. These equations are derived based on using either Cowell's method (position/velocity vectors) or Guass' method (orbital elements). In this table, the matrix $\mathbf{T}_{\mathrm{I}}^{\mathrm{LVLH}}$ denotes the direction cosine matrix of the LVLH coordinate frame with respect to the ECI coordinate frame, while $s(\cdot)$ $=\sin (\cdot)$ and $c(\cdot)=\cos (\cdot)$.

\section{Linear time-varying relative model}

In this section, a linear time-varying (LTV) high fidelity model is obtained to describe the relative motion dynamics. This model is derived based on two main assumptions. The first assumption is that the relative distance between the chaser and the target vehicles is much less than the orbital radius of target. The second assumption is that the main disturbance accelerations that affect both vehicles are the gravitational acceleration and the atmospheric drag acceleration. Based on these assumptions, all terms mentioned in the general relative dynamic expression (Equation (1)) are expanded up to only the first order to obtain the newly proposed model. Table 3 summarizes the procedures that have been followed to obtain this model. In this table, the linear time varying model is reduced to the following form

Table 1. Orbit Model Methods Summary

- Cowell's Method

$$
\begin{aligned}
& \ddot{\boldsymbol{R}}=\mathbf{f} \\
& \mathbf{f}=\mathbf{f}_{\mathrm{g}}+\mathbf{f}_{\mathrm{a}}+\mathbf{f}_{\mathrm{c}}+\mathbf{f}_{\mathrm{w}} \\
& \mathbf{f}_{\mathrm{g}}=\boldsymbol{a}_{t w o-b o d y}+\boldsymbol{a}_{J_{2}} \\
& \boldsymbol{a}_{\text {two-body }}=-\left(\frac{\mu}{R^{3}}\right) \boldsymbol{R}
\end{aligned}
$$

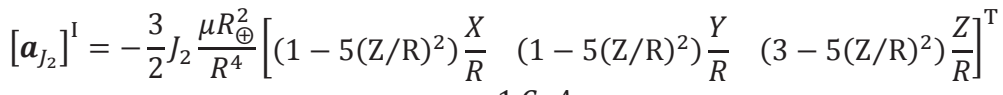

$$
\begin{aligned}
& {\left[\mathbf{f}_{\mathrm{a}}\right]^{\mathrm{I}}=-\frac{1}{2} \frac{C_{D} A}{m} \rho V \boldsymbol{V}}
\end{aligned}
$$

- Guass' Method

$$
\begin{aligned}
& \frac{d a}{d t}=\frac{2 a^{2}}{H}\left[e \mathrm{~s}_{f} \boldsymbol{a}_{x}+\frac{p}{R} \boldsymbol{a}_{y}\right] \\
& \frac{d e}{d t}=\frac{1}{H}\left\{p \mathrm{~s}_{f} \boldsymbol{a}_{x}+\left[(p+R) \mathrm{c}_{f}+R e\right] \boldsymbol{a}_{y}\right\} \\
& \frac{d i}{d t}=\frac{R \mathrm{c}_{\omega+f}}{H} \boldsymbol{a}_{z} \\
& \frac{d \Omega}{d t}=\frac{R s_{\omega+f}}{H s_{i}} \boldsymbol{a}_{z} \\
& \frac{d \omega}{d t}=\frac{1}{H e}\left\{-p \mathrm{c}_{f} \boldsymbol{a}_{x}+(p+R) \mathrm{s}_{f} \boldsymbol{a}_{y}\right\}-\frac{R \mathrm{~s}_{\omega+f} \mathrm{c}_{i}}{H \mathrm{~s}_{i}} \boldsymbol{a}_{z} \\
& \frac{d f}{d t}=\frac{H}{R^{2}}+\frac{1}{H e}\left[p \mathrm{c}_{f} \boldsymbol{a}_{x}-(p+R) \mathrm{s}_{f} \boldsymbol{a}_{y}\right] \\
& {[\boldsymbol{a}]^{\mathrm{LVLH}}=\left[\boldsymbol{a}_{J_{2}}+\mathbf{f}_{\mathrm{a}}+\mathbf{f}_{\mathrm{c}}+\mathbf{f}_{\mathrm{w}}\right]^{\mathrm{LLH}}} \\
& {\left[\boldsymbol{a}_{J_{2}}\right]^{L V L H}} \\
& =-\frac{3}{2} J_{2} \frac{\mu R_{\oplus}^{2}}{R^{4}}\left[1-3 \sin ^{2} i \sin ^{2}(\omega+f) \quad \sin ^{2} i \sin 2(\omega+f) \quad \sin 2 i \sin (\omega+f)\right]^{\mathrm{T}} \\
& {\left[\mathbf{f}_{\mathrm{a}}\right]^{\mathrm{LVLH}}=-\frac{1}{2} \frac{C_{D} A}{m} \rho V \boldsymbol{V}^{\mathrm{LVLH}}} \\
& \boldsymbol{V}^{\mathrm{LVLH}}=\frac{H}{p}\left[\begin{array}{lll}
e \sin f & p / R & 0
\end{array}\right]^{\mathrm{T}}
\end{aligned}
$$




$$
\dot{x}=A x
$$

where $\boldsymbol{x}$ is the state vector. This model can be used to approximate the time varying state transition matrix by expanding the time invariant exponential matrix solution in a Taylor series to the fourth order as follows.

$$
\phi_{k}=e^{A \Delta t} \approx \boldsymbol{I}+\boldsymbol{A} \Delta t+\frac{\boldsymbol{A}^{2} \Delta t^{2}}{2 !}+\frac{\boldsymbol{A}^{3} \Delta t^{3}}{3 !}+\frac{\boldsymbol{A}^{4} \Delta t^{4}}{4 !}
$$

This matrix is used in the next section as a part of the extended Kaman filter to propagate the states forward in time and to compute the filter parameters.

For close proximity operations, a proportional-derivative (PD) controller is employed for the translation control. The translation control algorithm computes the required continuous thrust $\mathbf{f}_{c}$, based on the previous linear model, to track the desired trajectory specified by the following guidance algorithm.

$$
\begin{aligned}
\mathbf{U}_{\Delta V}=\mathbf{f}_{\mathbf{c}} & =\boldsymbol{K}_{\boldsymbol{\rho}} \boldsymbol{\delta} \boldsymbol{\rho}+\boldsymbol{K}_{\dot{\boldsymbol{\rho}} \boldsymbol{\boldsymbol { \rho }}} \\
\boldsymbol{\delta} \boldsymbol{\rho} & =\boldsymbol{\rho}_{\text {des }}-\widehat{\boldsymbol{\rho}} \\
\boldsymbol{\delta} \dot{\boldsymbol{\rho}} & =\dot{\boldsymbol{\rho}}_{\text {des }}-\widehat{\dot{\boldsymbol{\rho}}}
\end{aligned}
$$

The proportional and derivative control gains $\boldsymbol{K}_{\boldsymbol{\rho}}$ and $\boldsymbol{K}_{\dot{\boldsymbol{\rho}}}$, which are determined based on the desired natural frequency $\omega_{\rho}$ and damping ratio $\varsigma_{\rho}$ of the translational control system.

$$
\boldsymbol{K}_{\boldsymbol{\rho}}=\omega_{\rho}^{2} \boldsymbol{I}_{3 \times 3} \quad \boldsymbol{K}_{\dot{\boldsymbol{\rho}}}=2 \omega_{\rho} \zeta_{\rho} \boldsymbol{I}_{3 \times 3}
$$

Variables $\boldsymbol{\rho}_{\text {des }}$ and $\dot{\boldsymbol{\rho}}_{\text {des }}$ are the desired relative position and relative velocity, respectively, to be tracked by the chaser vehicle, and they are defined by the guidance algorithms. It is worth noting that the equivalent continuous velocity increment $\Delta V$, based on the continuous thrust, can be approximated for small $\Delta \mathrm{t}$ to be

$$
\Delta \boldsymbol{V} \approx \boldsymbol{U}_{\Delta \boldsymbol{V}} \Delta t
$$

\section{Relative Navigation}

This section is concerned with the estimation of the position/velocity of the chaser relative to the target. The estimation is accomplished by using the range and angle measurements of the target from a LIDAR system in the LVLH frame of the chaser. The relative system uses an

Table 2. LVLH Coordinate Frame Orientation

- Given Inertial Position and Velocity

$$
\begin{gathered}
\mathbf{T}_{\mathrm{I}}^{\mathrm{LVLH}}=\left[\begin{array}{lll}
i_{x} & i_{y} & i_{z}
\end{array}\right]^{\mathrm{T}} \\
i_{x}=\frac{\boldsymbol{R}}{R}, \quad i_{z}=\frac{\boldsymbol{H}}{H}, \quad i_{y}=i_{z} \times i_{x} \\
\boldsymbol{\omega}=\left(\begin{array}{c}
R\left[\mathbf{f}_{z}\right]^{\mathrm{LVLH}} / H \\
0 \\
H / R^{2}
\end{array}\right), \quad \dot{\boldsymbol{\omega}}=\left(\begin{array}{c}
\frac{\left(\dot{R}\left[\mathbf{f}_{z}\right]^{\mathrm{LVLH}}+R\left[\dot{\mathbf{f}}_{z}\right]^{\mathrm{LVLH}}\right) H-R\left[\mathbf{f}_{z}\right]^{\mathrm{LVLH}} \dot{H}}{H^{2}} \\
0 \\
\frac{\dot{H} R-2 H \dot{R}}{R^{3}} \\
{[\dot{\mathbf{f}}]^{\mathrm{LVLH}}=\mathbf{T}_{\mathrm{I}}^{\mathrm{LVLH}}[\dot{\mathbf{f}}]^{\mathrm{I}}-\boldsymbol{\omega} \times \mathbf{T}_{\mathrm{I}}^{\mathrm{LVHH}}[\mathbf{f}]^{\mathrm{I}}} \\
\dot{R}=\frac{\boldsymbol{R} \cdot \boldsymbol{V}}{R}, \quad \boldsymbol{H}=\boldsymbol{R} \times \boldsymbol{V}
\end{array}\right.
\end{gathered}
$$

- Given Orbital Elements

$$
\begin{gathered}
\mathbf{T}_{\mathrm{I}}^{\mathrm{LVLH}}=\mathbf{T}^{3}(\omega+f) \mathbf{T}^{1}(i) \mathbf{T}^{3}(\Omega) \\
=\left[\begin{array}{ccc}
c_{\Omega} c_{\omega+f}-s_{\Omega} s_{\omega+f} c_{i} & s_{\Omega} c_{\omega+f}+c_{\Omega} s_{\omega+f} c_{i} & s_{\omega+f} s_{i} \\
-c_{\Omega} s_{\omega+f}-s_{\Omega} c_{\omega+f} c_{i} & -s_{\Omega} s_{\omega+f}+c_{\Omega} c_{\omega+f} c_{i} & c_{\omega+f} s_{i} \\
s_{\Omega} s_{i} & -c_{\Omega} s_{i} & c_{i}
\end{array}\right] \\
\boldsymbol{\omega}=\mathbf{T}^{3}(\omega+f) \mathbf{T}^{1}(i)\left[\begin{array}{l}
0 \\
0 \\
\dot{\Omega}
\end{array}\right]+\mathbf{T}^{3}(\omega+f)\left[\begin{array}{c}
d i / d t \\
d \\
0 \\
0
\end{array}\right]+\left[\begin{array}{c}
0 \\
0 \\
\dot{\omega}+\dot{f}
\end{array}\right]=\left(\begin{array}{cc}
s_{\omega+f} s_{i} \dot{\Omega}+c_{\omega+f} d i / d t \\
0 \\
c_{i} \dot{\Omega}+(\dot{\omega}+\dot{f})
\end{array}\right) \\
\mathbf{T}^{1}(\theta)=\left[\begin{array}{ccc}
1 & 0 & 0 \\
0 & c_{\theta} & s_{\theta} \\
0 & -s_{\theta} & c_{\theta}
\end{array}\right], \quad \mathbf{T}^{2}(\theta)=\left[\begin{array}{ccc}
c_{\theta} & 0 & -s_{\theta} \\
0 & 1 & 0 \\
s_{\theta} & 0 & c_{\theta}
\end{array}\right], \quad \mathbf{T}^{3}(\theta)=\left[\begin{array}{ccc}
c_{\theta} & s_{\theta} & 0 \\
-s_{\theta} & c_{\theta} & 0 \\
0 & 0 & 1
\end{array}\right]
\end{gathered}
$$


extended Kalman filter for the estimation purpose and PD controller for maneuver targeting. Orbital elements of the target are maintained by numerically propagating the Gauss' variational equations with $J_{2}$ and drag perturbations with respect to time. These orbital elements are used to compute the transformation matrix of the target vehicle with respect to the inertial frame and to assist in estimating LIDAR measurements.

\subsection{Extended Kalman Filter}

An extended Kalman filter is derived from the nonlinear models, as illustrated in the equations below [12].

$$
\dot{\boldsymbol{x}}=\boldsymbol{f}(\boldsymbol{x}, \boldsymbol{u}, \boldsymbol{t})+\boldsymbol{w}(\boldsymbol{t}), \quad \boldsymbol{w}(\boldsymbol{t}) \sim N(0, Q)
$$

$$
\tilde{\mathbf{z}}_{k}=\boldsymbol{h}\left(\boldsymbol{x}_{k}, \boldsymbol{t}\right)+\boldsymbol{v}_{k}, \quad \boldsymbol{v}_{k} \sim N\left(0, R_{k}\right)
$$

Here, the state vector $x$ can represent relative position/ velocity of the chaser as well as other parameters that need to be estimated for the use by other flight algorithms. The time derivatives of the states $\dot{x}$ are functions of the states, inputs, time, and additive process noise $\boldsymbol{w}$. This process noise is used to approximate the unmodeled disturbances and other random disturbances to the dynamics. The measurements $\tilde{\mathbf{z}}_{k}$ are modeled as functions of the states, time, and measurement noise $\boldsymbol{v}_{k}$. The process noise and measurement noise are normally distributed with zero mean and covariances $Q$ and $R_{k}$, respectively.

The following steps summarize the Kalman filter equations that are used to estimate the relative motion states, based on

Table 3. Relative Orbit Model Summary

- Relative Nonlinear Model

$$
\ddot{\boldsymbol{\rho}}=\left[\mathbf{f}_{c}\right]^{\mathrm{LVLH}}-\left[\mathbf{f}_{t}\right]^{\mathrm{LVLH}}-2 \boldsymbol{\omega} \times \dot{\boldsymbol{\rho}}-\boldsymbol{\omega} \times \boldsymbol{\omega} \times \boldsymbol{\rho}-\dot{\boldsymbol{\omega}} \times \boldsymbol{\rho}
$$

- Linear Time Varying Model

$$
\begin{aligned}
& -2 \omega \times \dot{\rho}-\omega \times \omega \times \rho-\dot{\omega} \times \rho \\
& =\left[\begin{array}{cccccc}
\omega_{z}^{2} & \dot{\omega}_{z} & -\omega_{x} \omega_{z} & 0 & 2 \omega_{z} & 0 \\
-\dot{\omega}_{z} & \omega_{x}^{2}+\omega_{z}^{2} & \dot{\omega}_{x} & -2 \omega_{z} & 0 & 2 \omega_{x} \\
-\omega_{x} \omega_{z} & -\dot{\omega}_{x} & \omega_{x}^{2} & 0 & -2 \omega_{x} & 0
\end{array}\right]\left(\begin{array}{c}
x \\
y \\
z \\
\dot{x} \\
\dot{y} \\
\dot{z}
\end{array}\right) \\
& {\left[\mathbf{f}_{c}\right]^{\mathrm{LVLH}}-\left[\mathbf{f}_{t}\right]^{\mathrm{LVLH}} \approx\left[\Delta \mathbf{f}_{\mathrm{g}}+\Delta \mathbf{f}_{\mathrm{a}}\right]^{\mathrm{LVLH}}} \\
& {\left[\Delta \boldsymbol{a}_{t w o-b o d y}\right]^{\mathrm{LVLH}} \approx \frac{\mu}{R^{3}}\left(\begin{array}{ccc}
2 & 0 & 0 \\
0 & -1 & 0 \\
0 & 0 & -1
\end{array}\right)\left(\begin{array}{l}
x \\
y \\
z
\end{array}\right)} \\
& {\left[\Delta \boldsymbol{a}_{J_{2}}\right]^{\mathrm{LVLH}}} \\
& \approx-\frac{3}{2} J_{2} \frac{\mu R_{\oplus}^{2}}{R^{5}}\left[\begin{array}{ccc}
-4\left(1-3 i_{x_{3}}^{2}\right) & -8 i_{x_{3}} i_{y_{3}} & -8 i_{x_{3}} i_{z_{3}} \\
-8 i_{x_{3}} i_{y_{3}} & 1-7 i_{x_{3}}^{2}+2\left(1-i_{z_{3}}^{2}\right) & 2 i_{y_{3}} i_{z_{3}} \\
-8 i_{x_{3}} i_{z_{3}} & 2 i_{y_{3}} i_{z_{3}} & 3-5 i_{x_{3}}^{2}-2\left(1-i_{z_{3}}^{2}\right)
\end{array}\right]\left(\begin{array}{l}
x \\
y \\
z
\end{array}\right) \\
& {\left[\Delta \mathbf{f}_{\mathrm{a}}\right]^{\mathrm{LVLH}} \approx\left[\begin{array}{ccc}
C_{c} \omega_{z} R & 0 & 0 \\
0 & C_{c} \omega_{z} R & 0 \\
0 & 0 & C_{c} \omega_{z} R
\end{array}\right]\left(\begin{array}{c}
\dot{x} \\
\dot{y} \\
\dot{z}
\end{array}\right)} \\
& \dot{\boldsymbol{x}}=\boldsymbol{A} \boldsymbol{x}, \quad \boldsymbol{x}=\left[\begin{array}{llllll}
x & y & z & \dot{x} & \dot{y} & \dot{z}
\end{array}\right]^{\mathrm{T}} \\
& A=\left[\begin{array}{ll}
A_{11} & A_{12} \\
A_{21} & A_{22}
\end{array}\right] \\
& A_{11}=0_{3 \times 3}, \quad A_{12}=I_{3 \times 3} \\
& A_{21}=\left[\begin{array}{ccc}
\omega_{z}^{2}+2 C_{1}-4 C_{2}\left(1-3 i_{x_{3}}^{2}\right) & \dot{\omega}_{z}-8 C_{2} i_{x_{3}} i_{y_{3}}-C_{c} \omega_{z}^{2} R & -\omega_{x} \omega_{z}-8 C_{2} i_{x_{3}} i_{z_{3}} \\
-\dot{\omega}_{z}-8 C_{2} i_{x_{3}} i_{y_{3}}+C_{c} \omega_{z}^{2} R & \left(\omega_{x}^{2}+\omega_{z}^{2}\right)-C_{1}+C_{2}\left(1-7 i_{x_{3}}^{2}+2\left(1-i_{z_{3}}^{2}\right)\right) & \dot{\omega}_{x}+2 C_{2} i_{y_{3}} i_{z_{3}}-C_{c} \omega_{x} \omega_{z} R \\
-\omega_{x} \omega_{z}-8 C_{2} i_{x_{3}} i_{z_{3}} & -\dot{\omega}_{x}+2 C_{2} i_{y_{3}} i_{z_{3}}+C_{c} \omega_{x} \omega_{z} R & \omega_{x}^{2}-C_{1}+C_{2}\left(3-5 i_{x_{3}}^{2}-2\left(1-i_{z_{3}}^{2}\right)\right)
\end{array}\right] \\
& \boldsymbol{A}_{\mathbf{2 2}}=\left[\begin{array}{ccc}
C_{c} \omega_{z} R & 2 \omega_{z} & 0 \\
-2 \omega_{z} & C_{c} \omega_{z} R & 2 \omega_{x} \\
0 & -2 \omega_{x} & C_{c} \omega_{z} R
\end{array}\right] \\
& C_{1}=\frac{\mu}{R^{3}}, \quad C_{2}=-\frac{3}{2} J_{2} \frac{\mu R_{\oplus}^{2}}{R^{5}}, \quad C_{c}=-\frac{1}{2} \frac{C_{D} A}{m} \rho
\end{aligned}
$$


minimizing mean square of error.

1. Enter prior state estimate $\widehat{\boldsymbol{x}}_{k}^{-}$and its error covariance $P_{k}^{-}$, and compute the Kalman gain

$$
K_{k}=P_{k}^{-} H_{k}^{T}\left(H_{k} P_{k}^{-} H_{k}^{T}+R_{k}\right)^{-1}
$$

2. Update state estimate by measurement $\mathrm{z}_{-} \mathrm{k}$

$$
\begin{gathered}
\hat{\mathbf{z}}_{k}^{-}=\boldsymbol{h}\left(\widehat{\boldsymbol{x}}_{k}^{-}\right) \\
\widehat{\boldsymbol{x}}_{k}=\widehat{\boldsymbol{x}}_{k}^{-}+K_{k}\left(\widetilde{\boldsymbol{z}}_{k}-\widehat{\boldsymbol{z}}_{k}^{-}\right)
\end{gathered}
$$

3. Compute error covariance for updated state estimate

$$
P_{k}=\left(\boldsymbol{I}-K_{k} H_{k}\right) P_{k}^{-}
$$

4. Project ahead

$$
\begin{gathered}
\widehat{\boldsymbol{x}}_{k+1}^{-}=\phi_{k} \widehat{\boldsymbol{x}}_{k} \\
P_{k+1}^{-}=\phi_{k} P_{k} \phi_{k}^{T}+Q_{k}
\end{gathered}
$$

In Equation (7), I denotes the $6 \times 6$ identity matrix, $\phi_{k}$ is the state transition matrix, and $H_{k}$ is the measurements of partial matrix that represents the sensitivity of the measurements to changes in the states.

By following the steps of References 12,14,15, and 19, the initial error covariance matrix $\mathrm{P}^{-}{ }_{0}$, which represents how accurately the initial relative position/velocity of the target is known, is given by

$$
P_{0}^{-}=\left[\begin{array}{cccccc}
\sigma_{x}^{2} & 0 & 0 & 0 & \varepsilon \sigma_{x} \sigma_{\dot{y}} & 0 \\
0 & \sigma_{y}^{2} & 0 & \varepsilon \sigma_{\dot{x}} \sigma_{y} & 0 & 0 \\
0 & 0 & \sigma_{z}^{2} & 0 & 0 & 0 \\
0 & \varepsilon \sigma_{\dot{x}} \sigma_{y} & 0 & \sigma_{\dot{x}}^{2} & 0 & 0 \\
\varepsilon \sigma_{x} \sigma_{\dot{y}} & 0 & 0 & 0 & \sigma_{\dot{y}}^{2} & 0 \\
0 & 0 & 0 & 0 & 0 & \sigma_{\dot{z}}^{2}
\end{array}\right]
$$

where $\sigma_{x}, \sigma_{y}$, and $\sigma_{z}$ denote the standard deviation uncertainties of the relative position components, and $\sigma_{\dot{x}}, \sigma_{\dot{y}}$, and $\sigma_{\dot{z}}$ are those of the relative velocity components. The coefficient $\varepsilon$ refers to the uncertainty correlation coupling between the relative position/velocity components in the LVLH coordinate frame, and it ranges between a positive and negative one.

The discrete process noise matrix $Q_{k}$ of the relative motion can be approximated by $[14,15]$

$$
Q_{k}=\left[\begin{array}{cccccc}
\sigma_{w_{x}}^{2}\left(\frac{\Delta t^{3}}{3}\right) & 0 & 0 & \sigma_{w_{x}}^{2}\left(\frac{\Delta t^{2}}{2}\right) & 0 & 0 \\
0 & \sigma_{w_{y}}^{2}\left(\frac{\Delta t^{3}}{3}\right) & 0 & 0 & \sigma_{w_{y}}^{2}\left(\frac{\Delta t^{2}}{2}\right) & 0 \\
0 & 0 & \sigma_{w_{z}}^{2}\left(\frac{\Delta t^{3}}{3}\right) & 0 & 0 & \sigma_{w_{z}}^{2}\left(\frac{\Delta t^{2}}{2}\right) \\
\sigma_{w_{x}}^{2}\left(\frac{\Delta t^{2}}{2}\right) & 0 & 0 & \sigma_{w_{x}}^{2}(\Delta t) & 0 & 0 \\
0 & \sigma_{w_{y}}^{2}\left(\frac{\Delta t^{2}}{2}\right) & 0 & 0 & \sigma_{w_{y}}^{2}(\Delta t) & 0 \\
0 & 0 & \sigma_{w_{z}}^{2}\left(\frac{\Delta t^{2}}{2}\right) & 0 & 0 & \sigma_{w_{z}}^{2}(\Delta t)
\end{array}\right]
$$

Here, $\sigma w_{x}, \sigma w_{y}$, and $\sigma w_{z}$ are the standard deviations for the random unmodeled acceleration disturbances that act on the relative motion, and $\Delta t$ is the sampling time period.

\subsection{Measurement Model}

One of the most important components of the filter is the measurement partial matrix $H_{k}$ which is used in updating the states and error covariance matrices with the measurements. Fig. 6 depicts the LIDAR line of sight measurements that are processed by the filter as follows: $\tilde{\rho}$ (range), $\tilde{\alpha}$ (azimuth angle), and $\tilde{\beta}$ (elevation angle). In proximity operations, the models for these measurements are as given below $[15,19]$.

$$
\begin{aligned}
& \tilde{\alpha}=\tan ^{-1}\left(\frac{i_{y}}{i_{x}}\right)+v_{\alpha}, \quad v_{\alpha} \sim N\left(0, \sigma_{\alpha}^{2}\right) \\
& \tilde{\beta}=\sin ^{-1}\left(i_{z}\right)+v_{\beta}, \quad v_{\beta} \sim N\left(0, \sigma_{\beta}^{2}\right) \\
& \tilde{\rho}=\rho+v_{\rho}, \quad v_{\rho} \sim N\left(0, \sigma_{\rho}^{2}\right)
\end{aligned}
$$

Variables $v_{\alpha}, v_{\beta}$, and $v_{\rho}$ are azimuth, elevation, and range measurement noises, respectively. In the absence of any other more suitable model of noise, these noises are assumed to be white, with zero mean and the standard deviations being equal to $\sigma_{\alpha}, \sigma_{\beta}$, and $\sigma_{\rho}$, respectively.

The unit line of sight vector can be written as a function of the relative position vector in the chaser LVLH reference coordinate frame as

$$
\boldsymbol{i}_{\text {los }}=\left(\begin{array}{c}
i_{x} \\
i_{y} \\
i_{z}
\end{array}\right)=\left(\begin{array}{c}
c_{\alpha} c_{\beta} \\
s_{\alpha} c_{\beta} \\
s_{\beta}
\end{array}\right)=-\frac{\rho}{\rho}=-\frac{1}{\sqrt{x^{2}+y^{2}+z^{2}}}\left(\begin{array}{l}
x \\
y \\
z
\end{array}\right)
$$

In particular, rewriting the measurement Eq. (10) in the standard form of the Kalman filter vector notation leads to

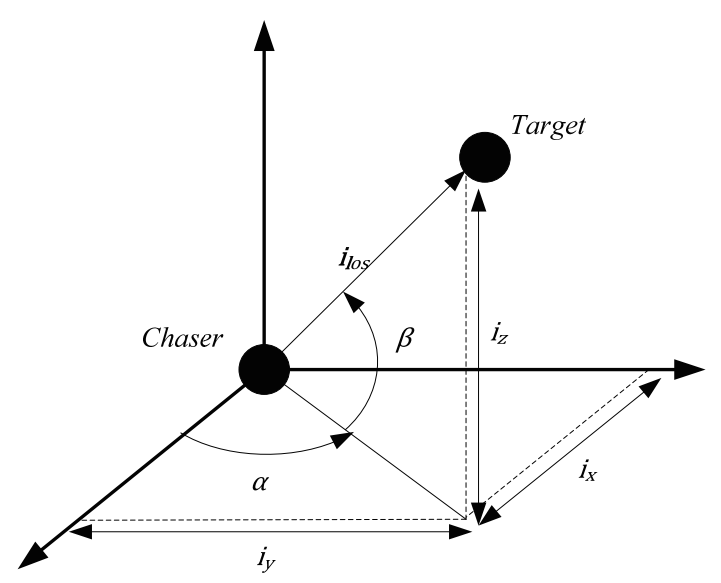

Fig. 3. Line of Sight Vector 


$$
\left(\begin{array}{c}
\tilde{\alpha} \\
\tilde{\beta} \\
\tilde{\rho}
\end{array}\right)=\left(\begin{array}{c}
\tan ^{-1}\left(\frac{i_{y}}{i_{x}}\right) \\
\sin ^{-1}\left(i_{z}\right) \\
\rho
\end{array}\right)+\left(\begin{array}{l}
v_{\alpha} \\
v_{\beta} \\
v_{\rho}
\end{array}\right)
$$

Now, the measurements for partial matrix $H_{k}$ is computed to be

$$
H_{k}=\left.\frac{\partial \boldsymbol{h}}{\partial x}\right|_{k}=\left[\begin{array}{cccccc}
\frac{-\hat{y}}{\hat{x}^{2}+\hat{y}^{2}} & \frac{\hat{x}}{\hat{x}^{2}+\hat{y}^{2}} & 0 & 0 & 0 & 0 \\
\frac{\hat{x} \hat{z}}{\rho^{2}\left(\hat{x}^{2}+\hat{y}^{2}\right)^{1 / 2}} & \frac{\hat{y} \hat{z}}{\rho^{2}\left(\hat{x}^{2}+\hat{y}^{2}\right)^{1 / 2}} & -\frac{\left(\hat{x}^{2}+\hat{y}^{2}\right)^{1 / 2}}{\rho^{2}} & 0 & 0 & 0 \\
\frac{\hat{x}}{\rho} & \frac{\hat{y}}{\rho} & \frac{\hat{z}}{\rho} & 0 & 0 & 0
\end{array}\right]_{k}
$$

and the associated measurement noise covariance matrix for the Kalman gain computations is

$$
R_{k}=\left[\begin{array}{ccc}
\sigma_{\alpha}^{2} & 0 & 0 \\
0 & \sigma_{\beta}^{2} & 0 \\
0 & 0 & \sigma_{\rho}^{2}
\end{array}\right]
$$

\section{Simulation Examples}

The key metrics of the analysis fall into three main categories. The first is navigation performance, which is how well the states are estimated by the filter. This metric is measured by the navigation error, the difference between the true states and the filtered states. The second is trajectory control performance, which is a measure of how closely the chaser vehicle is able to follow the guidance algorithms. The third is the fuel performance, or $\Delta \boldsymbol{V}$ usage, and it is computed based on the linear model developed in previous section.

The preceding guidance, navigation, and control algorithms are illustrated now through different examples. Initial conditions for simulation are listed in Tables 4 and 5 .

A Simulink model is built using MATLAB software to demonstrate the closed-loop guidance transfer of the chaser to approach and/or to depart from the target vehicle in any orbit, either circular or elliptic, given the uncertain initial conditions, noisy measurements, and limited dynamics. This model consists of three main parts, guidance, navigation, and control, and it is based on the closed loop GN\&C system block diagram shown in Fig. 4. The proposed LTV is used in the design of navigation filter and maneuver targeting of the guidance system. The required control thrust is produced based on a PD closed loop GN\&C system.

Table 4. Navigation Filter Parameters

\begin{tabular}{|c|c|}
\hline \multicolumn{1}{|c|}{ Parameter } & Value \\
\hline $\begin{array}{l}\text { Initial Relative Position and } \\
\text { Velocity Uncertainties }\end{array}$ & $\begin{array}{c}\sigma_{x}=\sigma_{y}=\sigma_{z}=33.3 \mathrm{~m} \\
\sigma_{\dot{x}}=\sigma_{\dot{y}}=\sigma_{\dot{z}}=0.01 \mathrm{~m} / \mathrm{s}\end{array}$ \\
\hline Process Noise & $\begin{array}{l}\sigma_{w_{x}}=\sigma_{w_{y}}=5 \times 10^{-4} \mathrm{~m} / \mathrm{s}^{3 / 2} \\
\sigma_{w_{z}}=5 \times 10^{-6} \mathrm{~m} / \mathrm{s}^{3 / 2}\end{array}$ \\
\hline Measurements Noise & $\sigma_{\alpha}=\sigma_{\beta}=0.06 \mathrm{deg}, \sigma_{\rho}=0.5 \mathrm{~m}$ \\
\hline Controller Parameters & $\omega_{\rho}=1 / 50 \mathrm{~s}^{-1}, \zeta_{\rho}=0.7$ \\
\hline Simulation Step & $0.1 \mathrm{~s}$ \\
\hline Measurements Update & $1 \mathrm{~Hz}$ \\
\hline
\end{tabular}

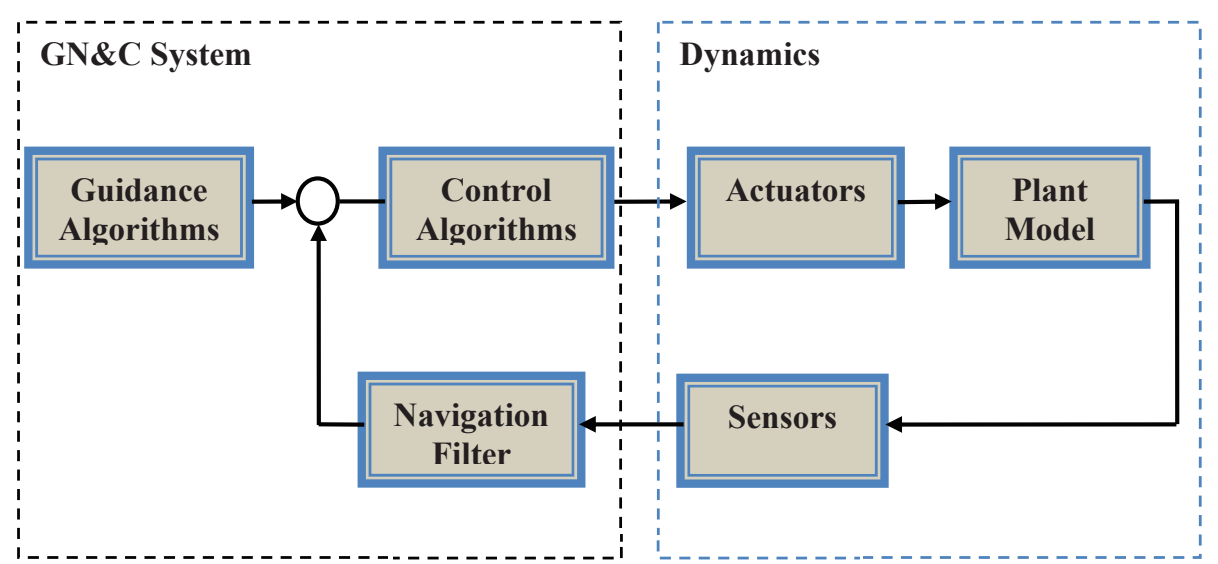

Fig. 4. Closed Loop GN\&C System 
Table 5. Vehicles Orbital Elements

\begin{tabular}{|c|c|c|}
\hline Parameter & Target & Chaser \\
\hline$a, \mathrm{~km}$ & 6723.2576 & 6723.2576 \\
\hline$e$ & 0.1 & 0.1 \\
\hline$i, \mathrm{deg}$ & 51.6467 & 51.6467 \\
\hline$\Omega, \mathrm{deg}$ & 188.0147 & 188.0147 \\
\hline$\omega, \mathrm{deg}$ & 174.3022 & 174.3022 \\
\hline$f, \mathrm{deg}$ & 270.0882 & 270.0832 \\
\hline
\end{tabular}

In order to evaluate the accuracy of the proposed LTV model, compared to other analytical closed form solution such as TH model, a simulation test case has been considered.

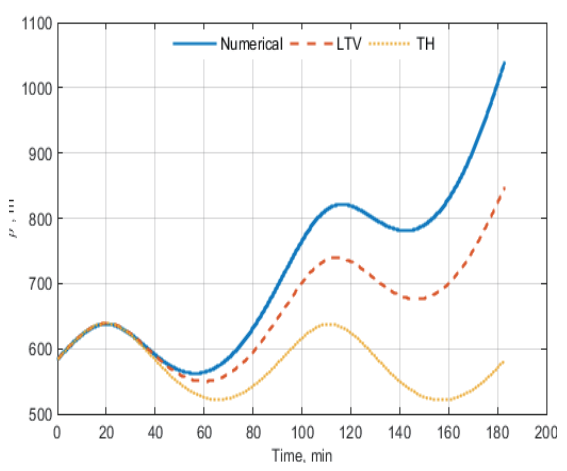

Fig. 5. High Fidelity LTV vs. TH vs. Full Nonlinear Numerical models
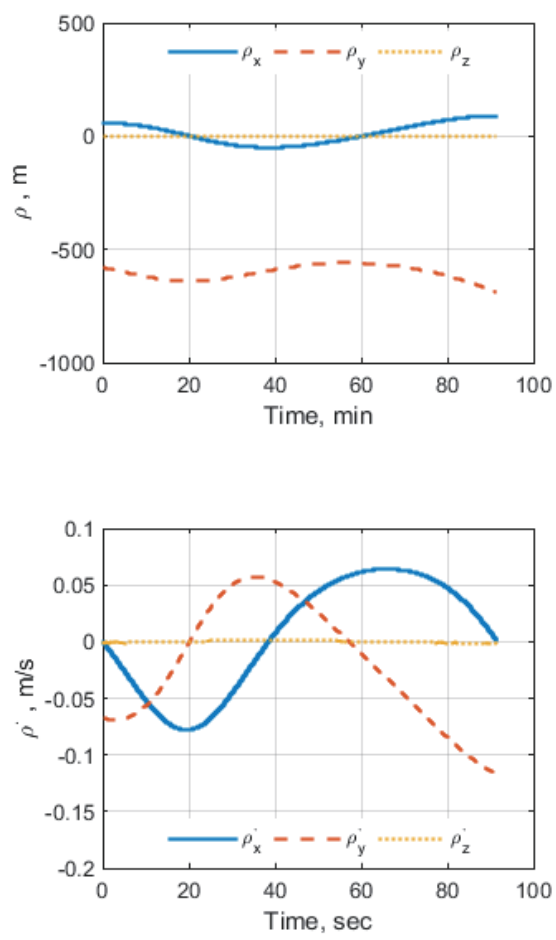

Fig. 6. Relative Motion Without $\Delta \mathrm{V}$
Fig. 5 shows the time history of the magnitude of relative position/relative velocity for the full nonlinear numerical, linear time varying (LTV), and linear analytical TH models of the chaser with respect to the target in low Earth eccentric orbit. It can be seen from the simulations that the proposed LTV model is more accurate than TH model over the time.

The performance of the navigation system is shown in Figs. 6 and 7. In this case, the thrusters are off, and both the target and chaser vehicles are in the same neighborhood (see Table 5). Fig. 6 shows the relative position/relative velocity between the vehicles during simulation. Fig. 7 depicts how accurately the navigation system can estimate the chaser's relative position/velocity. From this figure, the filter is able to converge within a few seconds, and the relative position/
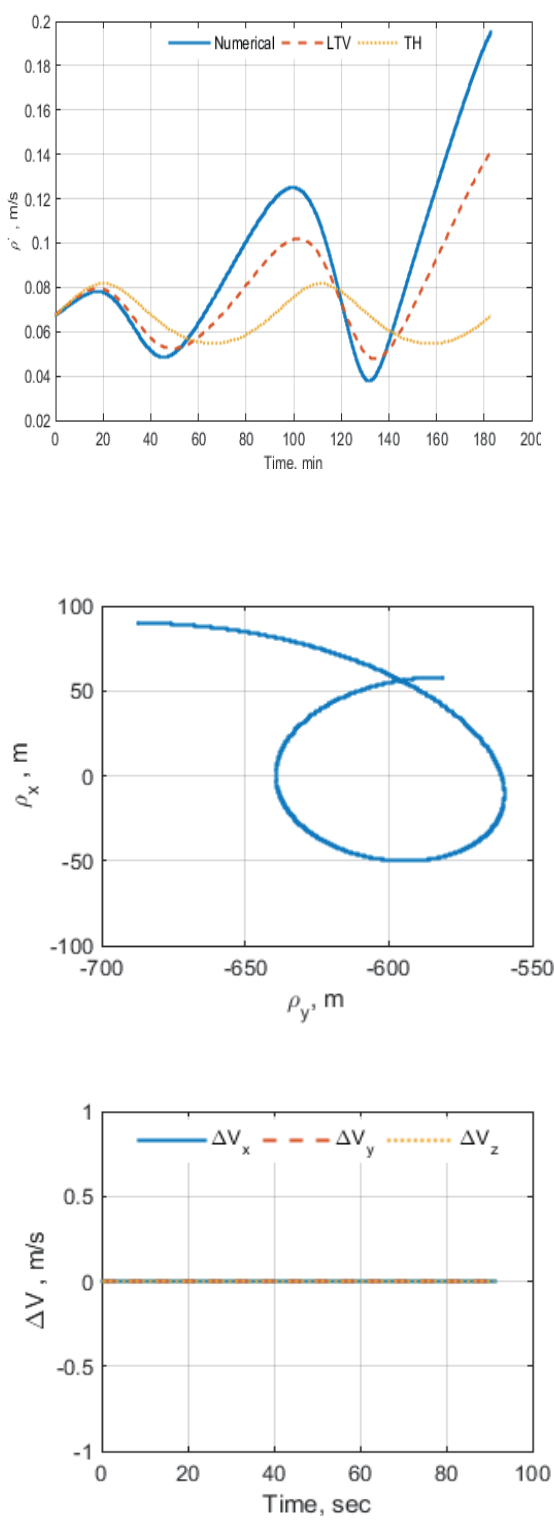
velocity can be accurately estimated within $0.5 \mathrm{~m}$ and 0.005 $\mathrm{m} / \mathrm{s}$, respectively.

Now simulation is presented for two types of glideslope
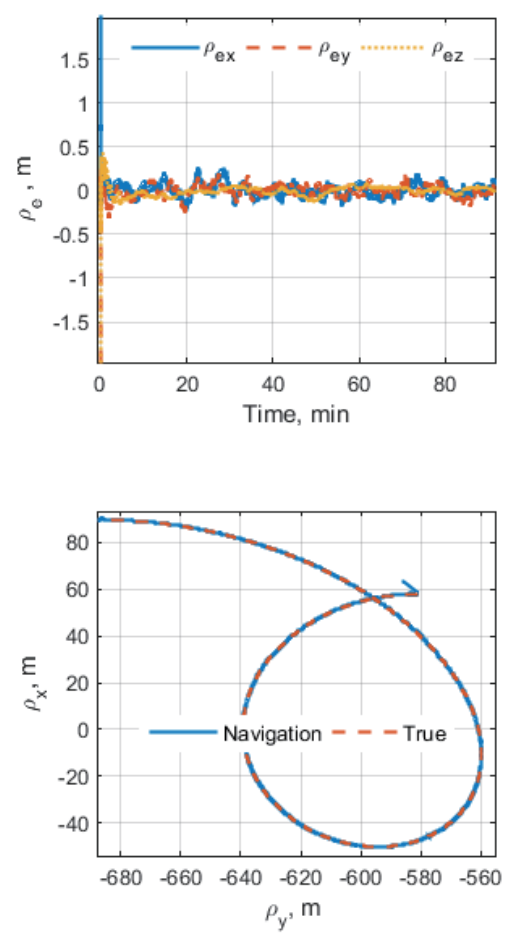

Fig. 7. Relative Motion Navigation Performance Without $\Delta V$
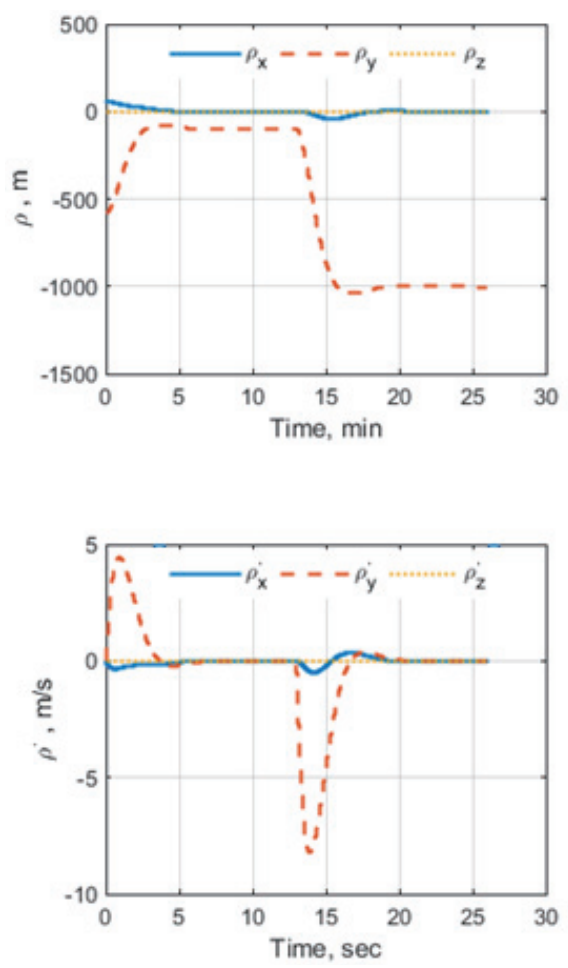

Fig. 8. Relative Motion Inbound/Outbound Scenario using PD Control trajectories, considering an eccentric orbit of the target. First, the inbound glideslope, in which the chaser is approaching the target vehicle, is shown in Figs. 8 and 9. The chaser is
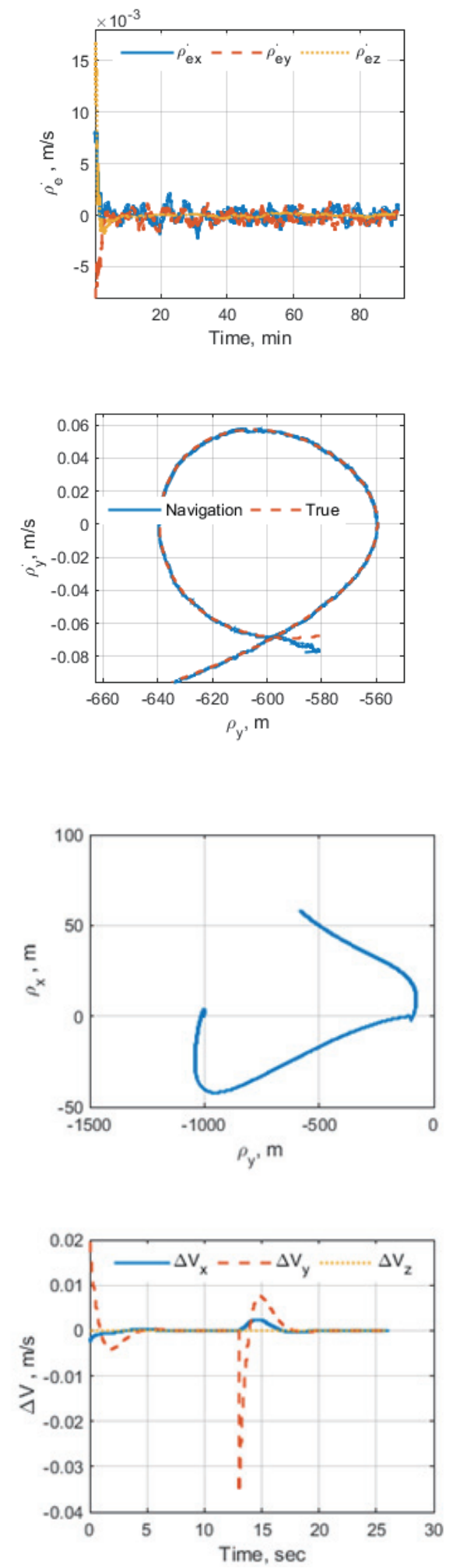
located at station [58 -580 0] $\mathrm{m}$, from which it is commanded to approach the target at $[0-1000] \mathrm{m}$. The relative position/ velocity errors and $\Delta \boldsymbol{V}$ continuous burns of the chaser are also shown in these figures. Second, the outbound glideslope is presented in the same figures in which the chaser is departing from the target. In these figures, the chaser starts to depart away from $100 \mathrm{~m}$ behind the target, leading to a new location of $1000 \mathrm{~m}$ behind the target. The corresponding performances of guidance, navigation, and control for the outbound accelerating glideslope are also shown in these figures. Each segment of the glideslope is followed by 3 minutes of station keeping, in which no thrust force is applied. From these figures, it is obvious that the PD design approach is successful in tracking a specific guidance trajectory.

The continuous thrust $\Delta \boldsymbol{V}$ is calculated by using the estimated relative position/velocity, either from the Kalman filter or from the knowledge of initial conditions, and not with the true relative position/velocity of the chaser. As such, the chaser is not expected to reach its intended place exactly, but in the neighborhood thereof. Aided by the sensors, the initial estimation errors subside to an optimal level determined by the ratio of the process noise matrix $Q_{k}$ and the measurement noise matrix $R_{k}$ defined earlier. Due to the active range and angle measurements from the LIDAR system and relatively small measurement errors, the true and the estimated relative position/velocity states are
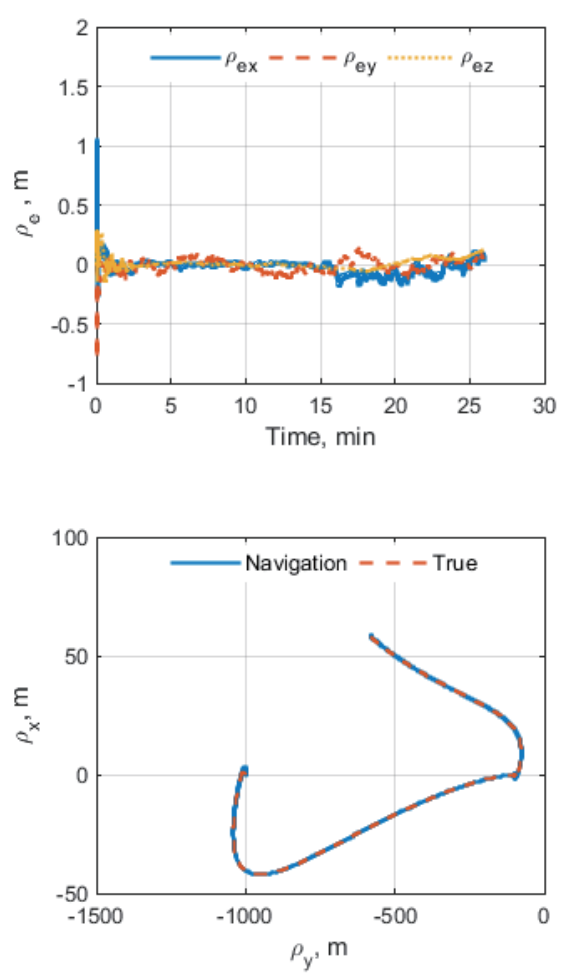

Fig. 9. Navigation and Control Performance Scenario using PD Control almost indistinguishable, as seen in the previous figures during the steady state.

\section{Conclusion}

The results of this study indicate that the proposed linear model is clearly effective in estimating the relative position/ velocity and controlling the relative trajectory. This model is not restricted to circular orbits, but it can be used as well for eccentric orbits. Furthermore, by using this model, simple guidance algorithms for glideslope are developed to autonomously approach and depart from a target vehicle. The relative navigation in this study utilizes range, azimuth, and elevation measurements, as well as an extended Kalman filter. However, uncertainties like measurement biases and sensor misalignments are not considered here. In addition, an analyst must consider the attitude dynamics of chaser and target, their attitude determination, and attitude control of the chaser, in order to fire the thrusters in the right direction. These topics and others will be addressed in the future.

\section{References}

[1] Fehse, W., Automated Rendezvous and Docking of
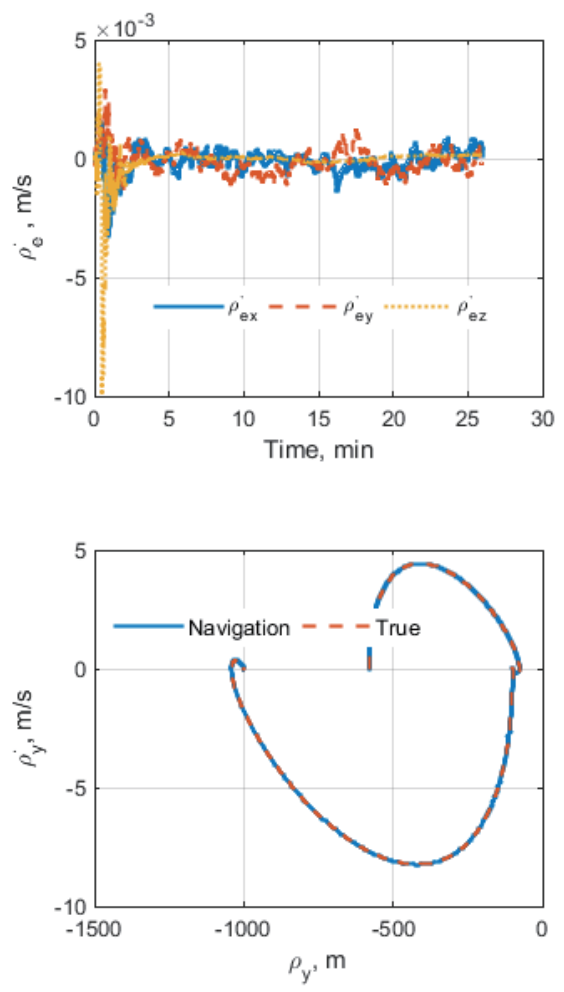
Spacecraft, 1st ed., Cambridge University Press, Cambridge, United Kingdom, 2003.

[2] Woffinden, D. C. and Geller, D. K., "Navigating the Road to Autonomous Orbital Rendezvous," Journal of Spacecraft and Rockets, Vol. 44, No. 4, 2007, pp. 898-909.

[3] Clohessy, W. H. and Wiltshire, R. S., "Terminal Guidance System for Satellite Rendezvous," Journal of the Astronautical Sciences, Vol. 27, No. 9, 1960, pp. 653-678.

[4] Tschauner, J. and Hempel, P., "Rendezvous zu einem in Elliptischer Bahn Umlaufenden Ziel," Acta Astronautica, Vol. 11, 1965, pp. 104-109.

[5] Yamanaka, K. and Ankersen, F., "New State Transition Matrix for Relative Motion on an Arbitrary Elliptical Orbit," Journal of Guidance, Control, and Dynamics, Vol. 25, No. 1, 2002, pp. 60-66.

[6] Carter, T. E., "State Transition Matrices for Terminal Rendezvous Studies: Brief Survey and New Example," Journal of Guidance, Control, and Dynamics, Vol. 21, No. 1, 1998, pp. 148-155.

[7] Melton, R. G., "Time-Explicit Representation of Relative Motion between Elliptical Orbits," Journal of Guidance, Control, and Dynamics, Vol. 23, No. 4, 2000, pp. 604-610.

[8] Broucke, R. A., "Solution of the Elliptic Rendezvous Problem with the Time as Independent Variable," Journal of Guidance, Control, and Dynamics, Vol. 26, No. 4, 2003, pp. 615-621.

[9] Inalhan, G., Tillerson, M. and How, J. P., "Relative Dynamics and Control of Spacecraft Formation in Eccentric Orbits," Journal of Guidance, Control, and Dynamics, Vol. 25, No. 1, 2002, pp. 48-58.

[10] Sengupta, P. and Vadali, S. R., "Relative Motion and the Geometry of Formations in Keplerian Elliptic Orbits," Journal of Guidance, Control, and Dynamics, Vol. 30, No. 4, 2007, pp. 953-964.

[11] Okasha, M. and Newman, B., "Relative Motion and
Autonomous Rendezvous in Keplerian Elliptic Orbits," AIAA 2010-7593, Proceedings of the AIAA Guidance, Navigation, and Control Conference, Toronto, Ontario, Canada, August, 2010 .

[12] Brown, R. G. and Hawag, P., Introduction to Random Signals and Applied Kalman Filtering, 3rd ed., John Wiley \& Son Inc., New York, New York, 1997.

[13] Jenkins, S. C. and Geller, D. K., "State Estimation and Targeting for Autonomous Rendezvous and Proximity Operations," AAS 07-316, Proceedings of the AIAA/AAS Astrodynamics Specialists Conference, Mackinac Island, Michigan, August 2007.

[14] Woffinden, D. C., "On-Orbit Satellite Inspection Navigation and $\Delta \mathrm{v}$ Analysis," M.S. Thesis, Dept. of Aeronautics and Astronautics, Massachusetts Institute of Technology, Cambridge, MA, 2004.

[15] Hablani, H. B., Tapper, M. L. and Dana-Bashian, D. J., "Guidance and Relative Navigation for Autonomous Rendezvous in a Circular Orbit," Journal of Guidance, Control, and Dynamics, Vol. 25, No. 3, 2002, pp. 553-562.

[16] Junkins, J. L., Kim, S., Crassidis, L., Cheng, Y. and Fosbury, A. M., "Kalman Filtering for Relative Spacecraft Attitude and Position Estimation," AIAA 2005-6087, Proceedings of the AIAA Guidance, Navigation, and Control Conference, San Francisco, California, August, 2005.

[17] Vallado, D. A., Fundamentals of Astrodynamics and Applications, 2nd ed., Microcosm Press, El Segundo, California, 2001.

[18] Schaub, H. and Junkins, J. L., Analytical Mechanics of Space Systems, American Institute of Aeronautics and Astronautics, Reston, Virginia, 2003.

[19] Woffinden, D. C. and Geller, D. K., "Relative AnglesOnly Navigation and Pose Estimation for Autonomous Orbital Rendezvous," Journal of Guidance, Control, and Dynamics, Vol. 30, No. 5, 2007, pp. 1455-1469. 